\title{
Evaluation of the effects of the combination of BMP-2-modified BMSCs and PRP on cartilage defects
}

\author{
SHIQIANG RUAN, JIANG DENG, LING YAN and WENLIANG HUANG \\ Department of Orthopaedics Surgery, The First People's Hospital of Zunyi, Zunyi, Guizhou 563003, P.R. China
}

Received July 1, 2017; Accepted July 20, 2018

DOI: 10.3892/etm.2018.6776

\begin{abstract}
Articular cartilage is avascular and aneural, and has limited capacity for self-regeneration when injured. Tissue engineering has emerged as a promising approach in repairing cartilage defects. To improve the therapy of cartilage healing, the present study investigated the efficacy of the combination of lentivirus-mediated bone morphogenetic protein-2 (BMP2) in bone marrow-derived stromal cells (BMSCs) and platelet-rich plasma (PRP) on cartilage and bone healing in a cartilage defect model using the rabbit knee. The BMSCs were harvested from New Zealand rabbits and transduced with lentivirus carrying BMP-2. Standard bone defects were introduced in the femoral groove of patellofemoral joints of 48 New Zealand rabbits. The cartilage defects were subjected to synthetic scaffold mosaicplasty with chitosan/silk fibroin/nanohydroxyapatite particles tri-component scaffolds soaked in BMSCs and PRP. After 16 weeks, the tissue specimens were assessed by micro-computed tomography (micro-CT) and macroscopic examination. The results showed that lentivirus-mediated BMP-2 and PRP increased the cell viability of the BMSCs, induced the expression of associated genes and enhanced osteogenic differentiation in vitro. In vivo, the expression of BMP-2 was observed for 16 weeks. The combination of BMP-2 and PRP treatment led to optimal results, compared with the other groups on micro-CT and gross observations. The results of the present study present a novel therapy using the lentivirus-mediated BMP-2 gene together with PRP for cartilage healing.
\end{abstract}

\section{Introduction}

Articular cartilage is avascular and aneural, and has limited capacity for self-regeneration when injured. Early intervention

Correspondence to: Dr Jiang Deng, Department of Orthopaedics Surgery, The First People's Hospital of Zunyi, 98 Fenghuang Road, Huichuan, Zunyi, Guizhou 563003, P.R. China

E-mail: dj30666@126.com

Key words: bone marrow stromal cells, bone morphogenetic protein-2, platelet rich plasma, tissue engineering, mosaicplasty, cartilage defect is essential to prevent further damage from spreading to peripheral healthy cartilage and joint surfaces, as osteoarthritis may occur in the injured joint, which eventually requires invasive treatment, for example, knee replacement (1).

Several clinical treatments for cartilage injuries have been developed, including microfracture (2), abrasion arthroplasty (3), autologous osteochondral transplant $(4,5)$, autologous chondrocyte implantation $(5,6)$ and prosthetic joint replacement (7). Autologous osteochondral transplant (mosaicplasty) is a technique that involves the transplantation of one or more cylindric osteochondral plugs harvested from the non-weight-bearing periphery of the femoral condyles towards the damaged cartilage site (8). Mosaicplasty has several advantages in terms of it being a single-stage and simple procedure, and being cost-effective. Mosaicplasty provides integration of subchondral bone into the host tissue and has been satisfied in numerous studies (9-14).

Tissue engineering has emerged as a promising approach in repairing cartilage defects as it provides an optimal strategy to develop a scaffold as a native cartilage-bone plug, which has similar structural and mechanical features as osteochondral tissue to facilitate the integration with host cartilage and underlying subchondral bone. The principles of tissue engineering involve three major components, including biocompatible scaffolds, the utilization of cell sources, and cell signaling molecules (15).

Chitosan (CS), silk fibroin (SF), and nanohydroxyapatite particles (nHA) are commonly used biomaterials due to their safety and biological compatibility (16-18). It has been found that, compared with bi-component scaffolds, the CS/SF/nHA tri-component scaffold has higher compressive strength and efficiency for inducing cell proliferation, which makes it a favorable scaffold for tissue engineering and regeneration (19).

Mesenchymal stem cells (MSCs) have been widely used as a potential cell source in several studies and clinical trials $(20,21)$. The high capacity of in vitro expansion of bone marrow-derived stromal cells (BMSCs) is an attractive source of cells for cartilage tissue engineering $(22,23)$. In addition, BMSCs are progenitor cells of osteoblasts and are essential for the maintenance of bone quality and quantity (24). It has been increasingly suggested that BMSCs contribute to rapid healing in a variety of reconstructive and restorative surgical procedures (25).

Bone morphogenetic protein (BMP) is an important molecule for facilitating the recovery of bone and cartilage by 
inducing the differentiation of mesenchymal osteoprogenitors and promoting osteoblastic maturation and function $(26,27)$. Of all BMPs, BMP-2 is the most potent bone growth factor and has been widely used for inducing BMSC differentiation into osteoblastic cells (28-30). BMP-2 protein therapy is a useful therapy that has been used in the treatment of various animal bony defect models (31-35). However, the limitation of a short half-life and high cost associated with large dose requirements in clinical situations cannot fully satisfy clinical requirements, which restricts the application of these exogenous proteins (36). Various vectors have been established for delivering genes in stem cells efficiently (37-39). Among these, lentivirus mediated gene transduction has been found to be effective for the long-term and stable expression of BMP-2 $(31,32,40,41)$.

A novel treatment, platelet-rich plasma (PRP), is a safe solution for simulating a natural repair process which contains a high concentration of platelets and a variety of growth factors (42). PRP has been used on BMSCs to assist in bone tissue regeneration. The combination of PRP and BMSCs has shown promising results on cartilage bone recovery in animal experiments $(43,44)$.

To optimize the treatment of cartilage defects, the present study investigated the effect of the combination of lentivirus-mediated BMP-2-modified BMSCs and PRP on articular cartilage injury of the rabbit knee following treatment with mosaicplasty. It was found that cartilage defects were successfully repaired with BMP-2-transduced BMSCs/PRP at 16 weeks post-surgery. This combination may provide a promising therapy for cartilage and bone healing.

\section{Materials and methods}

Rabbit BMSC culture. The animal experiments were performed at The Third Military Medical University (Chongqing, China), and were approved by the Laboratory Animal Welfare and Ethics Committee of the Third Military Medical University. The BMSCs were purchased from Cyagen Biosciences, Inc. (Santa Clara, CA, USA; cat. no. RBXMX-01001). The cells were plated with the initial seeding in a $100-\mathrm{mm}$ dish and cultured with Dulbecco's modified Eagle's medium (DMEM, Gibco, Thermo Fisher Scientific, Inc., Waltham, MA, USA) supplemented with $10 \%$ fetal bovine serum (Thermo Fisher Scientific, Inc.) in standard culture conditions of $37^{\circ} \mathrm{C}$ and $5 \%$ humidified $\mathrm{CO}_{2}$. The cells were maintained for 3 days prior to the first medium change. When the cells reached $90 \%$ confluency on days 7-9, the adherent cells were trypsinized and passaged approximately at a 1:3 split. The identity and mulitpotent differentiation ability along osteogenic, chondrogenic and adipogenic lineages was confirmed by Cyagen Biosciences, Inc. Cells in passage three were used for transduction with lentivirus.

Construction of Lv-BMP-2 and lentiviral infection of BMSCs. The BMP-2 gene was amplified from Rabbit cDNA with primers containing NotI and BamHI restriction sites: Bmp-2-NotI, forward 5'-aaggaaaaaagcggccgcATGGTGGCCGGGACCCG CTG-3', Bmp-2-BamHI, reverse 5'-cgcggatccCTAGCGACA CCCACAACCCTCCAC-3'. Lv-BMP-2 was constructed by inserting the BMP-2 gene fragment into the pLV5-EF1A-GFP plasmid (Western Biotechnology, Inc., Chongqing, China). The lentivirus was produced by co-transfecting the pLV5-EF1A-GFP
(Lv-control or Lv-BMP-2) and helper plasmid PG-P1-VSVG, PG-P2-REV and PG-P3-RRE into 293T cells (Western Biotechnology, Inc.). The rabbit BMSCs at passage three were transduced with Lv-BMP-2 or Lv-control virus in the presence of $5 \mu \mathrm{g} / \mathrm{ml}$ polybrene. At $24 \mathrm{~h}$ post-transduction, the medium was replaced with fresh DMEM with $10 \%$ FBS. The BMSCs were cultured for $72 \mathrm{~h}$. GFP-positive cells were observed and infection efficiency was determined using an inverted fluorescence microscope.

PRP preparation (45). A 10-ml blood sample was harvested from the central auricular vein of rabbits from each group. The blood was transferred into an ethylene diamine tetraacetic acid vacutainers (BD Biosciences, Franklin Lakes, NJ, USA), and plasma was harvested by centrifugation for $10 \mathrm{~min}$ at $1,000 \mathrm{xg}$ at $22^{\circ} \mathrm{C}$. The supernatant was isolated and centrifuged for $20 \mathrm{~min}$ at $1,500 \mathrm{x} \mathrm{g}$ at $22^{\circ} \mathrm{C}$. The top layer was removed and the platelet-rich lower layer was collected for future use.

Construction of the osteochondral defect model. A total of 48 female New Zealand white rabbits (Jiangsu Zhenlin Biological Technology Co., Ltd., Jiangsu, China) aged 2-3 months old with a mean weight of $2.5 \mathrm{~kg}$ were housed in room at $26 \pm 3^{\circ} \mathrm{C}$ with a $12 \mathrm{~h}$ light/dark cycle. The rabbits were allowed ad libitum access to food and water. A full-thickness segment femoral cartilage defect (diameter $5 \mathrm{~mm}$, thickness $4 \mathrm{~mm}$ ) was generated through the articular cartilage and subchondral bone of the patellar groove (Fig. 1A) using a drill equipped with a 5-mm diameter drill bit. The $\mathrm{CS} / \mathrm{SF} / \mathrm{nHA}$ scaffold treated with or without PRP and BMSCs was then implanted to fill the cartilage defect on one knee (Fig. 1B). The rabbits were divided randomly into four groups: i) Untreated, ii) treated with BMSCs, iii) treated with Lv-control-BMSCs+PRP; iv) treated with Lv-BMP-2-BMSCs+PRP. A total of $10^{7} \mathrm{BMSCs}$ and/or $0.2 \mathrm{ml}$ PRP were used to treat different knees according to the grouping. The wound was then closed in layers. The animals were returned to their cages and allowed to move freely without joint immobilization. The rabbits were sacrificed at 16 weeks. Each cartilage defect area was evaluated macroscopically.

Scaffold preparation. The biphysic scaffold osteochondral defect repair were prepared according to our previously described procedures (19).

Reverse transcription-quantitative polymerase chain reaction (RT-qPCR) analysis. The cells from each group were collected and total RNA was extracted using TRIzol reagent (Invitrogen; Thermo Fisher Scientific, Inc.). The cDNA was then synthesized using a PrimeScript ${ }^{\circledR}$ 1st Strand cDNA Synthesis kit (Takara Biotechnology Co., Ltd., Dalian, China). A total of $1 \mathrm{mg}$ cDNA was used and RT-qPCR analysis was then performed using SYBR $^{\circledR}$ Premix Ex Taq ${ }^{\mathrm{TM}}$ (Takara Biotechnology Co., Ltd.) according to the manufacturer's protocol. The PCR conditions were as follows: Stage 1, pre-denaturation: $95^{\circ} \mathrm{C}$ for $4 \mathrm{~min}$; stage 2, PCR amplification: $95^{\circ} \mathrm{C}$ for $10 \mathrm{sec}, 60^{\circ} \mathrm{C}$ for $20 \mathrm{sec}$, $72^{\circ} \mathrm{C}$ for $30 \mathrm{sec}$ for a total of 40 cycles. Each experiment was performed in triplicate. The primers sequences for alkaline phosphatase (ALP), type I collagen and type II collagen are presented in Table I $(46,47)$. Relative mRNA expression was calculated using the $2^{-\Delta \Delta \mathrm{Ct}}$ method (48). 
Table I. Reverse transcription-quantitative polymerase chain reaction primer sequences.

Primer $\left(5^{\prime}-3^{\prime}\right)$

\begin{tabular}{lll}
\cline { 2 - 3 } Gene & \multicolumn{1}{c}{ Forward } & \multicolumn{1}{c}{ Reverse } \\
\hline ALP $(46)$ & GCACGACAATCGGGATGAAC & TCCAGCAGGACGGTCATCA \\
COL I $(47)$ & TGGCAAGAACGGAGATGACG & GCACCATCCAAACCACTGAA \\
COL II $(47)$ & CCACGCTCAAGTCCCTCAAC & AGTCACCGCTCTTCCACTCG \\
GAPDH & GGGTGGTGGACCTCATGGT & CGGTGGTTTGAGGGCTCTTA \\
\hline
\end{tabular}
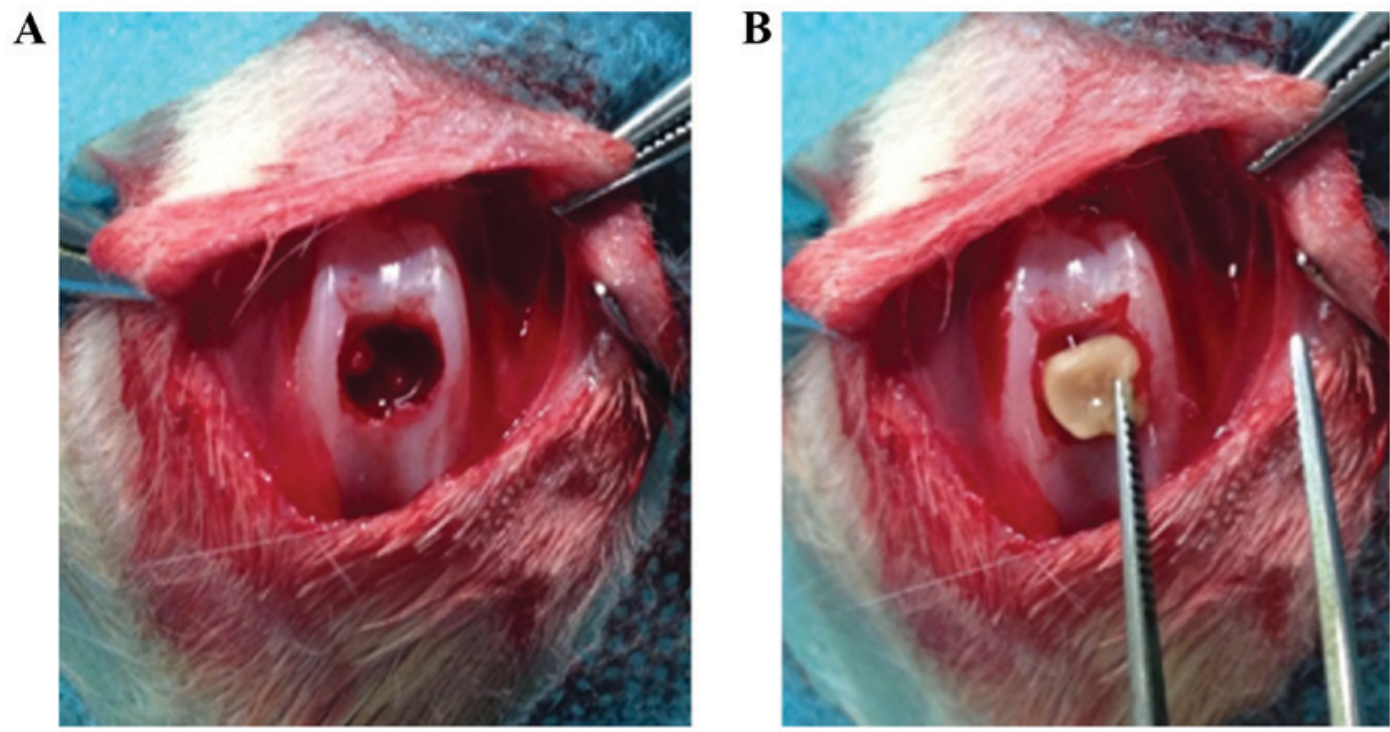

Figure 1. Construction of osteochondral defect model. A total of 48 New Zealand white rabbits were used. (A) A full-thickness segment femoral cartilage defect (diameter $5 \mathrm{~mm}$, thickness $4 \mathrm{~mm}$ ) was generated through the articular cartilage and subchondral bone of the patellar groove using a drill equipped with a 5-mm diameter drill bit. (B) A chitosan/silk fibroin/nanohydroxyapatite particle scaffold treated with or without platelet-rich plasma and bone marrow-derived stromal cells was implanted to fill the cartilage defect on one knee.

Western blot analysis. The cells from each group were washed with ice-cold phosphate-buffered saline (PBS) and lysed with lysis buffer containing $50 \mathrm{mM}$ Tris-HCL (pH 7.4), $0.1 \%$ sodium dodecyl sulfate (SDS), $150 \mathrm{mM} \mathrm{NaCl}$, and protease inhibitor cocktail (Sigma-Aldrich; Merck KGaA, Darmstadt, Germany). The protein concentration was determined using Pierce $^{\mathrm{TM}}$ BCA Protein Assay kit (Thermo Fisher Scientific, Inc.) according to the manufacturer's potocol. The sample was suspended in SDS buffer and boiled for 6 min. A total of $30 \mu \mathrm{g} /$ lane proteins were separated onto $30 \%$ SDS-PAGE gels for electrophoresis and then transferred onto a nitrocellulose membrane. The membranes were blocked in non-fat milk in Tris-buffered saline with Tween 20 (TBST) for $1 \mathrm{~h}$, and incubated with primary antibodies for BMP-2 (1:500; cat. no. bs-1012R; BIOSS, Beijing, China), SRY-box 9 (SOX9; 1:500; cat. no. HPA001758; Sigma-Aldrich; Merck KGaA) or $\beta$-actin (1:1,000; cat. no. MA5-15739; Thermo Fisher Scientific, Inc.) overnight at $4^{\circ} \mathrm{C}$. The membranes were washed with TBST three times and were incubated with goat anti-rabbit (cat. no. ab6721) and rabbit anti-mouse (cat. no. ab97046) secondary antibodies (both 1:1,000; Abcam, Cambridge, UK) for $1 \mathrm{~h}$ at room temperature. The bands were visualized by enhanced chemiluminescence detection reagents (Bio-Rad
Laboratories, Inc., Hercules, CA, USA) according to the manufacturer's protocol. The bands of western blot were quantified with ImageJ (ImageJ bundled with Java 1.8.0; National Institutes of Health, Bethesda, MD, USA) (49).

MTT assay. The cells were seeded $2 \times 10^{3}$ cells into 96 -well plates in triplicate. After $24 \mathrm{~h}$, the cells were washed twice with PBS and the MTT (Sigma; EMD Millipore) assays were performed. A total of $20 \mu \mathrm{l}$ MTT (5 g/l) was added to each well, and the cells were cultured for an additional $4 \mathrm{~h}$. The absorbance (A) values at $490 \mathrm{~nm}$ were determined with a microplate reader to determine cell viability.

Micro-computed tomography (CT) imaging. Specimens obtained at 16 weeks (three animals in each group) were examined with micro-CT (GE micro-CT system; GE Healthcare Life Sciences, Logan, UT, USA) for the analyses of bone and cartilage growth and regeneration.

Alizarin red staining. The cells were cultivated with osteogenetic induction solution $(0.1 \mu \mathrm{mol} / 1$ dexamethasone, $50 \mathrm{mg} / 1$ ascorbic acid and $10 \mathrm{mmol} / 1 \beta$-glycerophosphate) and cultured at $37^{\circ} \mathrm{C}$ and $5 \% \mathrm{CO}_{2}$. The medium was replaced every 3 days. 

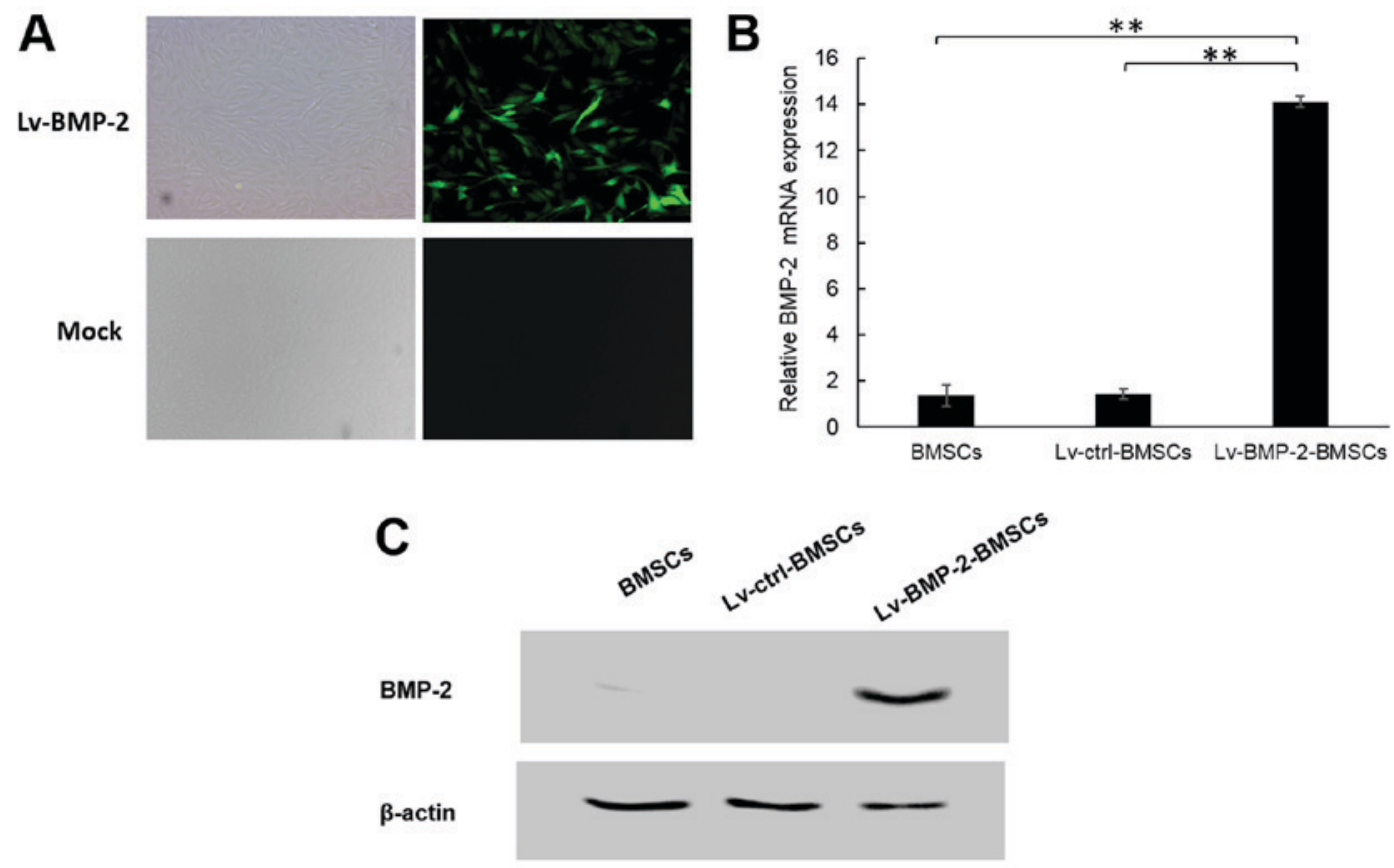

Figure 2. Lentivirus-mediated expression of BMP-2 in BMSCs. (A) At passage three, BMSCs were infected with Lv-BMP-2 at an MOI of 50 . After $72 \mathrm{~h}$, expression of GFP was examined by fluorescence microscopy. (B) mRNA expression of BMP-2 in BMSCs ( $\left.{ }^{* *} \mathrm{P}<0.01\right)$. (C) Protein expression of BMP-2 in BMSCs. BMP-2, bone morphogenetic protein-2; BMSCs, bone marrow-derived stromal cells; ctrl, control. Magnification, x200.

At 14 days post-induction, the cells were fixed with $95 \%$ ethanol for $10 \mathrm{~min}$, and incubated in $2 \%$ Alizarin red staining solution (Sigma-Aldrich; Merck KGaA) for 5 min. The staining buffer was removed and the cells were washed twice with PBS. Calcification deposits were identified under light microscopy.

Statistical analysis. The data were processed using SPSS statistical processing software (ver. 21.0; IBM SPSS, Armonk, NY, USA). The results are expressed as the mean \pm standard deviation. A unpaired Student's t-test was applied for statistical comparisons between two groups and one-way analysis of variance was applied among multiple groups. $\mathrm{P}<0.05$ was considered to indicate a statistically significant difference.

\section{Results}

Lentivirus mediated expression of BMP-2 in BMSCs. The BMSCs were transduced with lentivirus Lv-BMP-2 or Lv-control at an MOI of 50. According to fluorescence microscopy observation, the infection efficiency was $90 \%$ at $72 \mathrm{~h}$ post-transduction (Fig. 2A). To confirm the expression of BMP-2, RT-qPCR and western blot analyses were applied to detect the mRNA and protein levels of BMP-2 in BMSCs. As shown in Fig. 2B and C, the endogenous expression of BMP-2 in BMSCs was low, whereas the Lv-BMP-2 BMSCs expressed significantly higher BMP-2 compared with the Lv-control BMSCs and non-infected BMSCs at the mRNA and protein levels. This suggested that the Lv-BMP-2-modified BMSCs had been successfully established.

Combination of BMP-2 and PRP enhances the cell viability of BMSCs. The cell viability of cells was examined using an MTT assay. The absorbance value at $490 \mathrm{~nm}$ was significantly increased by infection with Lv-BMP-2 in BMSCs with PRP, compared with that in other groups (Fig. 3). It was also noted that PRP alone enhanced the cell viability of BMSCs, although the enhancement was not as marked as for the combination of BMP-2 and PRP.

Combination of BMP-2 and PRP results in enhanced expression of osteogenic-related genes in BMSCs. The impact of BMP-2 and PRP on the expression of osteogenic-related genes was then examined. ALP, and type I collagen are markers of osteogenic differentiation. In addition, type II collagen is known to modulate the chondrogenesis of MSCs and is important in the osteogenic differentiation of BMSCs (50). To investigate the capacity of osteogenic differentiation of BMP-2 BMSCs, the cells were cultured with osteogenesis induction medium, and total mRNA was isolated from the BMSCs from each group at day 7. The expression levels of ALP, type I collagen and type II collagen were determined by RT-qPCR analysis. The data showed that the lentivirus-mediated expression of BMP-2 in BMSCs upregulated the expression of ALP, type I collagen and type II collagen, compared with expression in the Lv-control group. Although incubation with PRP marginally increased the expression of these genes, the result was not statistically significant (Fig. 4).

Combination of BMP-2 and PRP induces higher osteogenic differentiation in BMSCs. Following 14 days of culture in osteogenesis induction medium, followed by Alizarin red staining, mineralised nodules were observed under the microscope. The mineralized nodules were more intense in the LvBMP-2-modified BMSCs + PRP group, compared with those in the other groups (Fig. 5A-D). Although BMP-2 or PRP alone marginally increased the osteogenic differentiation, LvBMP-2-modified BMSCs+PRP exerted the most marked enhancement of osteogenesis. 


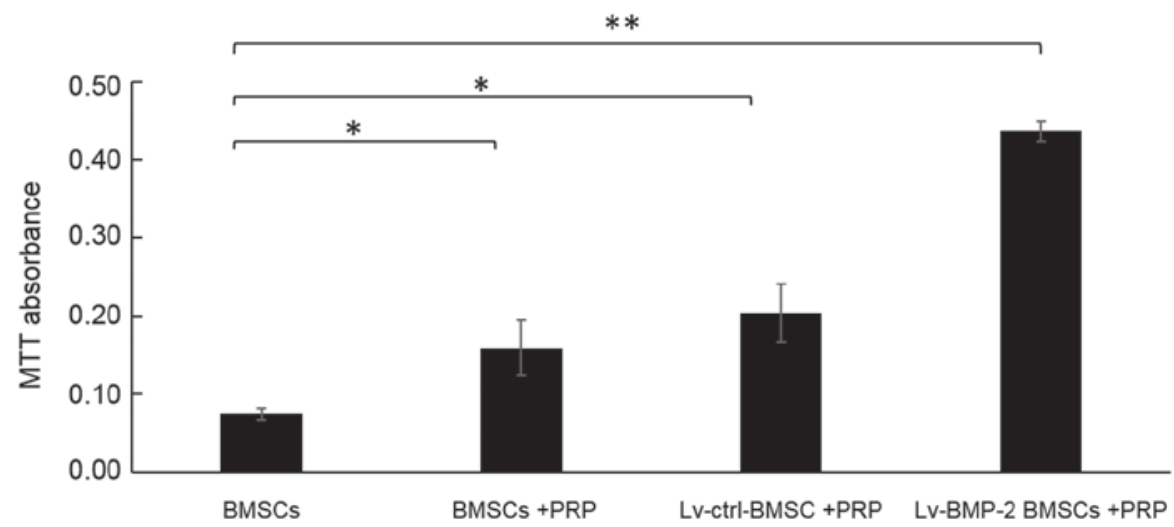

Figure 3. Combination of BMP-2 and PRP enhances the cell viability of BMSCs. An MTT assay was performed and the absorbance at $490 \mathrm{~nm}$ was determined to estimate changes in cell viability ( $\left.\mathrm{P}<0.05,{ }^{* *} \mathrm{P}<0.01\right)$. BMP-2, bone morphogenetic protein-2; BMSCs, bone marrow-derived stromal cells; PRP, platelet-rich plasma.

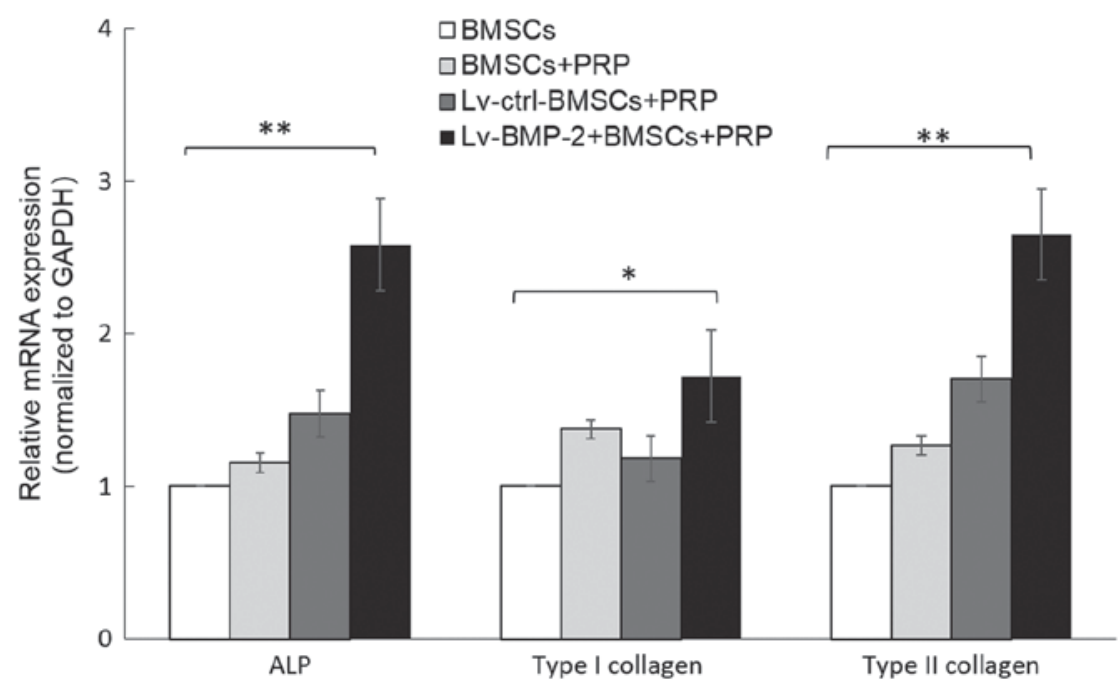

Figure 4. Combination of BMP-2 and PRP results in enhanced expression of osteogenic-related genes. Relative expression of ALP, type I collagen and type II collagen during the osteogenic differentiation of BMSCs. Cells were cultured with osteogenic induction medium, and total RNA was collected at 7 days. The mRNA expression levels of ALP, type I collagen and type II collagen were evaluated by reverse transcription-quantitative polymerase chain reaction analysis $\left({ }^{*} \mathrm{P}<0.05,{ }^{* * *} \mathrm{P}<0.01\right)$. BMP-2, bone morphogenetic protein-2; BMSCs, bone marrow-derived stromal cells; PRP, platelet-rich plasma; ctrl, control; ALP, alkaline phosphatase.

Combination of BMP-2 and PRP recovers cartilage defects completely. At day 0 , a full-thickness defect was created through the articular cartilage and subchondral bone of the patellar groove using a drill bit. The CS/SF/nHA scaffold was implanted to fill the cartilage defect. Subsequently, $10^{7}$ BMSCs and/or $0.2 \mathrm{ml} \mathrm{PRP} \mathrm{were} \mathrm{used} \mathrm{to} \mathrm{treated} \mathrm{different}$ knees according to grouping. The wound was then closed in layers. At 16 weeks, the cartilage defects were examined by macroscopic observation and micro-CT.

In the control group, the central regions of the defect sites were filled with a thin, rough reddish-brown tissue (Fig. 6), the patellar fossa was significantly larger than that of other groups (Figs. 6 and 7), indicating that cartilage repair remained limited and incomplete at week 16 . The defects in the Lv-BMP-2 BMSCs and Lv-control BMSCs+PRP groups were similar on gross observation (Figs. 6 and 7). Although a concavity was observed in the peripheral region of defects, the surface of cartilage and bone was shiny and smooth. In the BMP-2 BMSCs+PRP group, it was found that the surface region of the recovered cartilage was smooth and shiny, and covered with hyaline-like cartilage tissue (Fig. 6). No significant fossa was observed, suggesting that articular cartilage repair was complete. Taken together, the data showed that the combination of BMP-2-modified BMSCs with PRP had optimal effects on the recovery of cartilage and bone defects.

Lentivirus-mediated expression of BMP-2 is observed in BMSCs for at least 16 weeks. To confirm the expression of BMP-2 in cartilage defect region in rabbits, the proteins were isolated from articular cartilage and subchondral bone regions from each group at week 16. There was an increase of the overall expression of BMP-2 in tissues around the cartilage defect sites in the Lv-BMP-2+PRP group. It was also observed that PRP or Lv-BMP-2 alone enhanced the expression of BMP-2. SRY-box 9 (SOX9), a transcription factor that is important in cartilage development and formation was also detected. As shown in Fig. 8A and B, BMP-2 and PRP increased the expression of SOX9, which contributed to the 


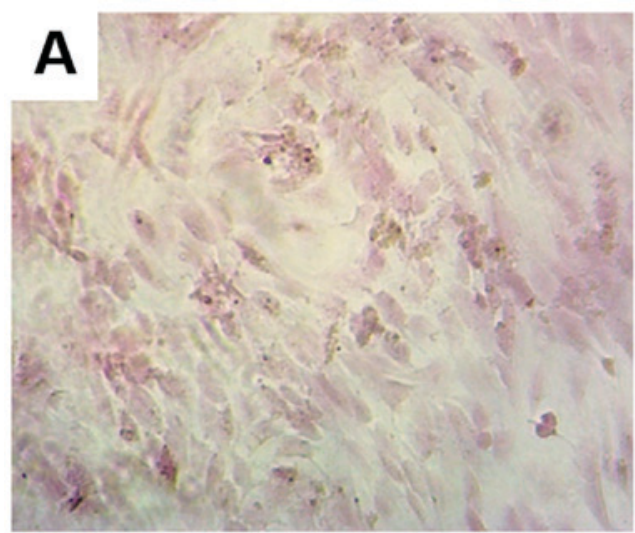

BMSCs

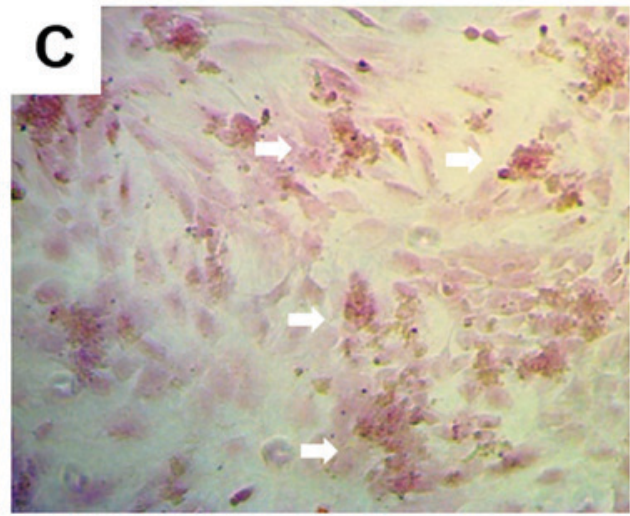

Lv-ctrl-BMSCs+PRP

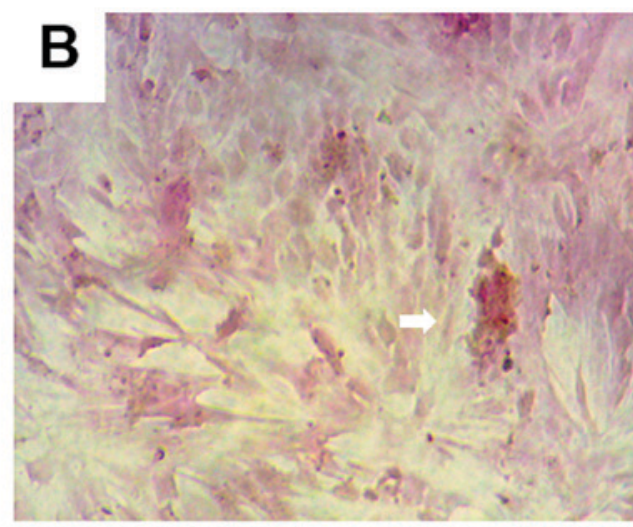

Lv-BMP-2-BMSCs

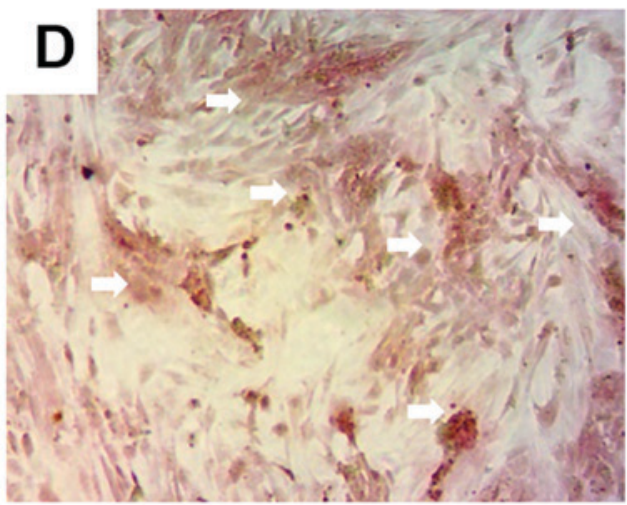

Lv-BMP-2-BMSCs+PRP

Figure 5. Osteogenic differentiation in BMSCs. Cells were cultured with osteogenesis induction medium for 14 days followed by Alizarin red staining. Mineralized nodules in the (A) BMSCs, (B) Lv-BMP-2 BMSCs, (C) Lv-ctrl BMSCs+PRP, and (D) Lv-BMP-2 BMSCs+PRP were observed under a microscope (magnification, x400). White arrows show mineralized calcium deposits. BMP-2, bone morphogenetic protein-2; BMSCs, bone marrow-derived stromal cells; PRP, platelet-rich plasma; ctrl, control.

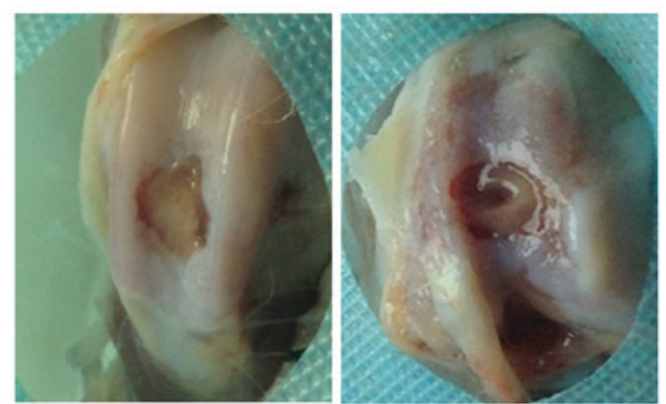

Control
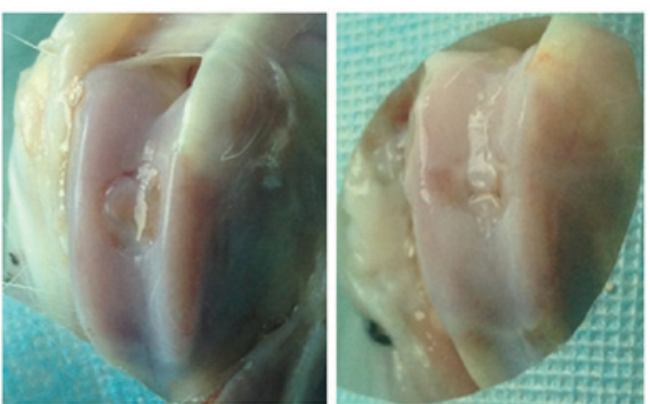

Lv-BMP-2-BMSCs
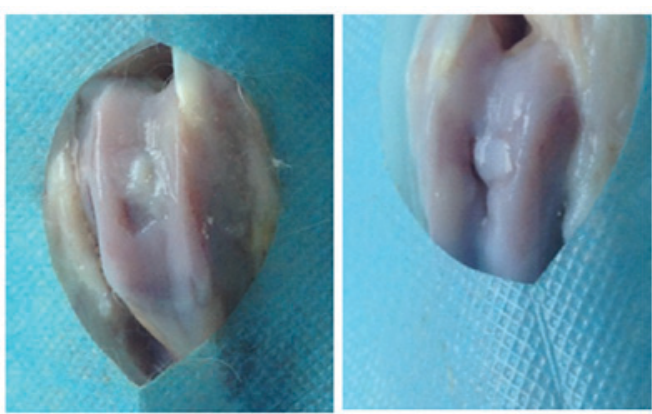

Lv-ctrl-BMSCs+PRP

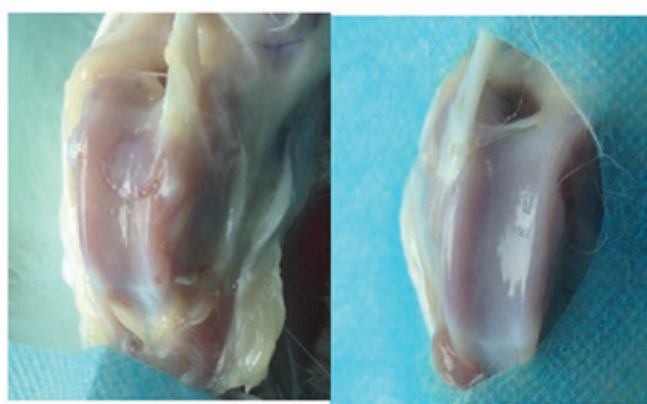

Lv-BMP-2-BMSCs+PRP

Figure 6. Gross observation of cartilage bone wounds. Representative gross data of cartilage wounds from each group at 16 weeks. BMP-2, bone morphogenetic protein-2; BMSCs, bone marrow-derived stromal cells; PRP, platelet-rich plasma; ctrl, control. 

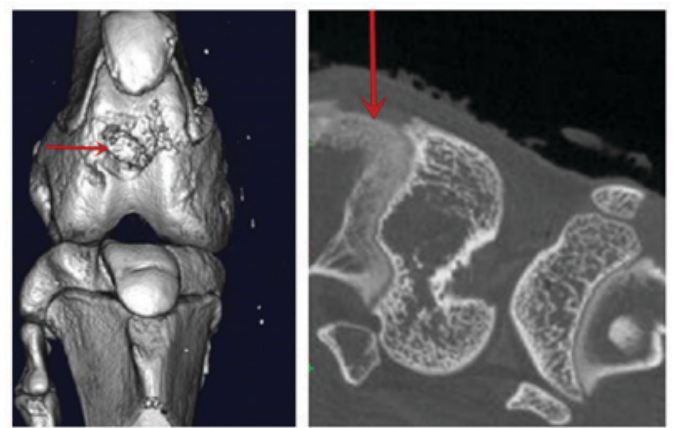

Control
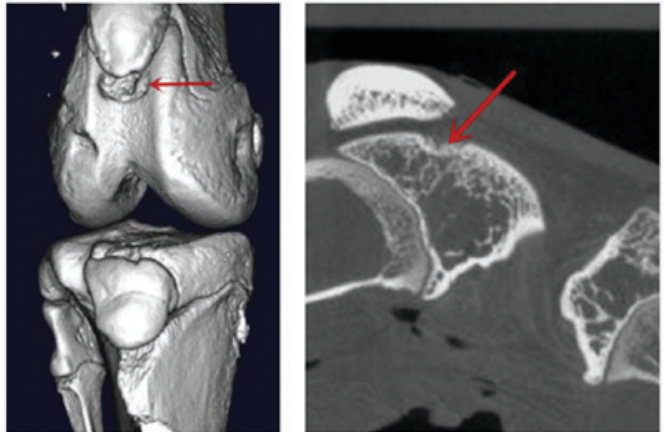

Lv-BMP-2-BMSCs
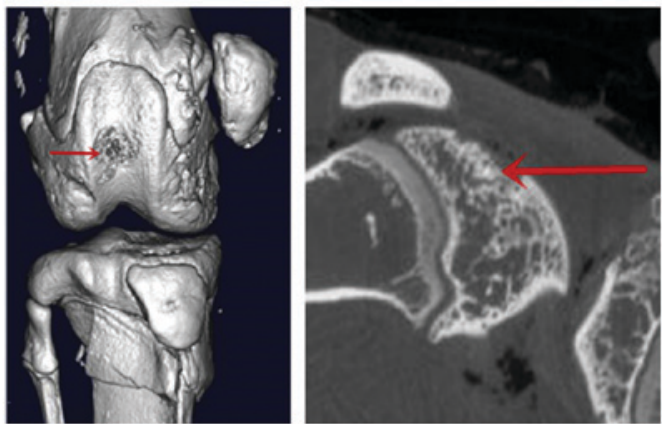

Lv-ctrl-BMSCs+PRP
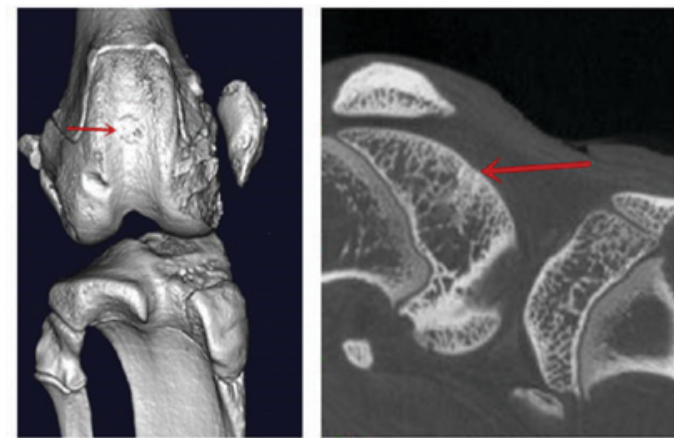

Lv-BMP-2-BMSCs+PRP

Figure 7. Micro-CT observation of cartilage bone wounds. Representative micro-CT of cartilage wounds from each group at 16 weeks. Red arrows indicate the cartilage defect. CT, computed tomography; BMP-2, bone morphogenetic protein-2; BMSCs, bone marrow-derived stromal cells; PRP, platelet-rich plasma; ctrl, control.
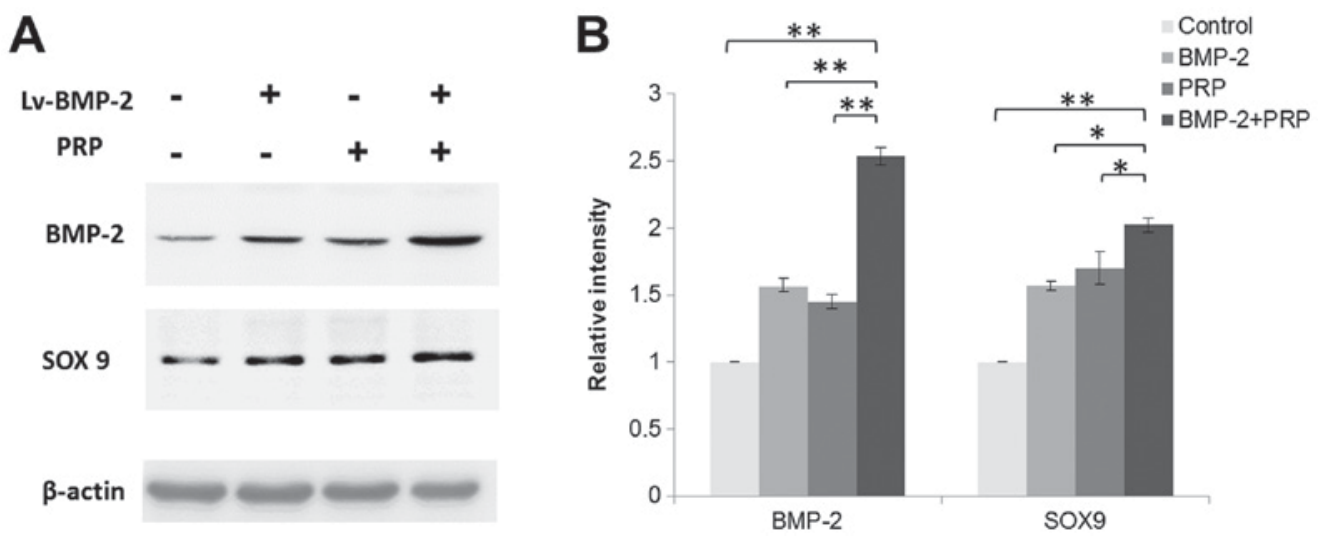

Figure 8. Lentivirus-mediated expression of BMP-2 in bone marrow-derived stromal cells is observed for at least 16 weeks. (A) Protein was isolated from articular cartilage and subchondral bone areas from each group in week 16. Western blot analysis was performed to detect the protein expression of BMP-2 and SOX9. (B) Relative expression of proteins normalized by $\beta$-actin ( $\mathrm{n}=3$; $\left.{ }^{*} \mathrm{P}<0.05,{ }^{* *} \mathrm{P}<0.01\right)$. BMP-2, bone morphogenetic protein-2; SOX9, SRY-box 9; PRP, platelet-rich plasma.

recovery of the cartilage defect in vivo. The data indicated that lentivirus-mediated expression of BMP-2 was present in BMSCs for at least 16 weeks, and the enhanced expression of BMP-2 combined with PRP treatment induced the expression of genes for cartilage and bone formation, which facilitated tissue regeneration in the rabbit knees.

\section{Discussion}

Articular cartilage has limited ability for self-regeneration, therefore, early intervention is important (1). MSCs have been widely used for tissue regeneration, particularly cartilage repair, owing to their potential for osteoregeneration and osteogenic differentiation $(20,21)$. There are three important components for successful tissue regeneration, namely, biocompatible scaffolds, cell sources, and cell signaling molecules (15). In the present study, the effect of the combination of a CS/SF/nHA tri-component scaffold, BMP-2-modified BMSCs and PRP on a rabbit cartilage defect model was investigated; the results showed that this combination provided promising results.

$\mathrm{CS}, \mathrm{SF}$ and nHA have been applied in various tissue engineering studies due to favourable biological compatibility (16-18). It has been found that a combination of two of these improved biocompatibility, enhanced mechanical strength, 
or reduced degradation $(16,17,51,52)$. Qi et al $(19)$, found that the CS/nHA/SF tri-component scaffold had all of the above advantages, making it suitable for the aim of the present study.

BMP-2 has been found to be important in bone formation and BMSC differentiation, compared with other BMP family members (28-30). However, its limitation of a short half-life and high cost prevents it from further application. With the assistance of lentivirus-mediated gene transduction, the present study showed that the expression of BMP-2 in BMSCs was sustained over at least 16 weeks, suggesting that it was effective for long-term and stable expression of BMP-2 (40).

PRP is another important factor for cartilage defects used in the present study. PRP provides various cell signaling molecules, which contribute to healing and growth, including platelet-derived growth factor, vascular endothelial growth factor, and transforming growth factor- $\beta 1$ (53). By using PRP, BMP-2-modified BMSCs exerted beneficial results on repairing cartilage defects in rabbit knees. Previously Bahmanpour et al (54), found that platelet-rich fibrin (PRF) had higher evaluation scores of full-thickness lesions in rabbits than PRP. The use of PRF, rather than PRP, to treat the cartilage defect in the model used in the present study may assist in identifying a more effective therapy.

This combination strategy has provided beneficial outcomes for tissue regeneration in several studies. For example, Lian et al (55), found that the combination of BMSCs and PRP assists in diabetic wound repair and regeneration. BMSCs transfected with BMP-2 also enhanced tendon-bone healing and improved bone regeneration in canine segmental ulnar defects $(56,57)$. The present study combined the advantages of several components for tissue regeneration and optimized the cartilage defect therapy in a rabbit model.

Taken together, the data obtained in the present study suggested that the combination of BMP-2 and PRP enhanced the efficiency of cartilage and bone repair and provided a novel promising therapy for tissue engineering.

\section{Acknowledgements}

Not applicable.

\section{Funding}

The present study was financially supported by the Science and Technology Plan Projects in Guizhou Province [grant no. (2016)1420] and the National Natural Science Foundation of China (grant no. 81660367).

\section{Availability of data and materials}

The datasets generated and analysed during the present study are available from the corresponding author on reasonable request.

\section{Authors' contributions}

JD was involved in the design of the current study. SR and LY performed all experiments. WH performed the statistical analyses. All authors participated in manuscript preparation and were involved in the discussion of the results. All authors have read and approved the final manuscript.

\section{Ethics approval and consent to participate}

The animal experiments were approved by the Laboratory Animal Welfare and Ethics Committee of the Third Military Medical University.

\section{Patient consent for publication}

Not applicable.

\section{Competing interests}

The authors confirm that they have no competing interests.

\section{References}

1. Nehrer S, Chiari C, Domayer S, Barkay H and Yayon A: Results of chondrocyte implantation with a fibrin-hyaluronan matrix: A preliminary study. Clin Orthop Relat Res 466: 1849-1855, 2008.

2. Steadman JR, Rodkey WG and Rodrigo JJ: Microfracture: Surgical technique and rehabilitation to treat chondral defects. Clin Orthop Relat Re S362-S369, 2001.

3. Bittberg M: Articular cartilage repair: An update on different clinical repair methods. Ortop Traumatol Rehabil 3: 235-243, 2001.

4. Mendicino RW, Catanzariti AR and Hallivis R: Mosaicplasty for the treatment of osteochondral defects of the ankle joint. Clin Podiatr Med Surg 18: 495-513, 2001.

5. Bentley G, Biant LC, Carrington RW, Akmal M, Goldberg A, Williams AM, Skinner JA and Pringle J: A prospective, randomised comparison of autologous chondrocyte implantation versus mosaicplasty for osteochondral defects in the knee. J Bone Joint Surg Br 85: 223-230, 2003.

6. Brittberg M, Tallheden T, Sjogren-Jansson B, Lindahl A and Peterson L: Autologous chondrocytes used for articular cartilage repair: An update. Clin Orthop Relat Res: (391 Suppl) S337-S348, 2001.

7. Hunziker EB: Articular cartilage repair: Basic science and clinical progress. A review of the current status and prospects. Osteoarthritis Cartilage 10: 432-463, 2002.

8. Hangody L, Rathonyi GK, Duska Z, Vasarhelyi G, Fules P and Módis L: Autologous osteochondral mosaicplasty. Surgical technique. J Bone Joint Surg Am 86-A (Suppl 1): S65-S72, 2004.

9. Ozturk A, Ozdemir MR and Ozkan Y: Osteochondral autografting (mosaicplasty) in grade IV cartilage defects in the knee joint: 2- to 7-year results. Int Orthop 30: 200-204, 2006.

10. Haklar U, Tuzuner T, Kocaoglu B and Guven O: Mosaicplasty technique in the treatment of osteochondral lesions of the knee. Acta Orthop Traumatol Turc 42: 344-349, 2008 (In Turkish).

11. Rose T, Craatz S, Hepp P, Raczynski C, Weiss J, Josten C and Lill H: The autologous osteochondral transplantation of the knee: Clinical results, radiographic findings and histological aspects. Arch Orthop Trauma Surg 125: 628-637, 2005.

12. Hangody L, Vasarhelyi G, Hangody LR, Sukosd Z, Tibay G, Bartha L and Bodó G: Autologous osteochondral grafting-technique and long-term results. Injury 39 (Suppl 1): S32-S39, 2008.

13. Emre TY, Ege T, Kose O, Tekdos Demircioglu D, Seyhan B and Uzun M: Factors affecting the outcome of osteochondral autografting (mosaicplasty) in articular cartilage defects of the knee joint: Retrospective analysis of 152 cases. Arch Orthop Trauma Surg 133: 531-536, 2013.

14. Hangody L, Dobos J, Balo E, Panics G, Hangody LR and Berkes I: Clinical experiences with autologous osteochondral mosaicplasty in an athletic population: A 17-year prospective multicenter study. Am J Sports Med 38: 1125-1133, 2010.

15. Langer R and Vacanti JP: Tissue engineering. Science 260: 920-926, 1993.

16. Bhardwaj $\mathrm{N}$ and Kundu SC: Silk fibroin protein and chitosan polyelectrolyte complex porous scaffolds for tissue engineering applications. Carbohydrate Polymers 85: 325-333, 2011. 
17. Bhardwaj N, Nguyen QT, Chen AC, Kaplan DL, Sah RL and Kundu SC: Potential of 3-D tissue constructs engineered from bovine chondrocytes/silk fibroin-chitosan for in vitro cartilage tissue engineering. Biomaterials 32: 5773-5781, 2011.

18. Ji WC, Zhang XW and Qiu YS: Selected suitable seed cell, scaffold and growth factor could maximize the repair effect using tissue engineering method in spinal cord injury. World J Exp Med 6: $58-62,2016$

19. Qi XN, Mou ZL, Zhang J and Zhang ZQ: Preparation of chitosan/silk fibroin/hydroxyapatite porous scaffold and its characteristics in comparison to bi-component scaffolds. J Biomed Mater Res A 102: 366-372, 2014

20. Garg P, Mazur MM, Buck AC, Wandtke ME, Liu J and Ebraheim NA: Prospective Review of Mesenchymal Stem Cells Differentiation into Osteoblasts. Orthop Surg 9: 13-19, 2017.

21. Fellows CR, Matta C, Zakany R, Khan IM and Mobasheri A: Adipose, bone marrow and synovial joint-derived mesenchymal stem cells for cartilage repair. Front Genet 7: 213, 2016.

22. Yamasaki S, Mera H, Itokazu M, Hashimoto Y and Wakitani S: Cartilage repair with autologous bone marrow mesenchymal stem cell transplantation: Review of preclinical and clinical studies. Cartilage 5: 196-202, 2014.

23. Potier E, Noailly J and Ito K: Directing bone marrow-derived stromal cell function with mechanics. J Biomech 43: 807-817, 2010

24. Chen Q, Shou P, Zheng C, Jiang M, Cao G, Yang Q, Cao J, Xie N, Velletri T, Zhang X, et al: Fate decision of mesenchymal stem cells: adipocytes or osteoblasts? Cell Death Differ 23: 1128-1139, 2016.

25. Schneider RK, Anraths J, Kramann R, Bornemann J, Bovi M, Knüchel R and Neuss S: The role of biomaterials in the direction of mesenchymal stem cell properties and extracellular matrix remodelling in dermal tissue engineering. Biomaterials 31: 7948-7959, 2010

26. Abe E: Function of BMPs and BMP antagonists in adult bone. Ann N Y Acad Sci 1068: 41-53, 2006.

27. Lochab AK and Extavour CG: Bone morphogenetic protein (BMP) signaling in animal reproductive system development and function. Dev Biol 427: 258-269, 2017.

28. Zhang Q, He QF, Zhang TH, Yu XL, Liu Q and Deng FL: Improvement in the delivery system of bone morphogenetic protein-2: A new approach to promote bone formation. Biomed Mater 7: 045002, 2012

29. Starman JS, Bosse MJ, Cates CA and Norton HJ: Recombinant human bone morphogenetic protein-2 use in the off-label treatment of nonunions and acute fractures: A retrospective review. J Trauma Acute Care Surg 72: 676-681, 2012.

30. Angle SR, Sena K, Sumner DR, Virkus WW and Virdi AS: Healing of rat femoral segmental defect with bone morphogenetic protein-2: A dose response study. J Musculoskelet Neuronal Interact 12 : 28-37, 2012 .

31. Hsu WK, Sugiyama O, Park SH, Conduah A, Feeley BT, Liu NQ, Krenek L, Virk MS, An DS, Chen IS and Lieberman JR: Lentiviral-mediated BMP-2 gene transfer enhances healing of segmental femoral defects in rats. Bone 40: 931-938, 2007.

32. Miyazaki M, Sugiyama O, Tow B, Zou J, Morishita Y, Wei F, Napoli A, Sintuu C, Lieberman JR and Wang JC: The effects of lentiviral gene therapy with bone morphogenetic protein-2-producing bone marrow cells on spinal fusion in rats. J Spinal Disord Tech 21: 372-379, 2008.

33. Jiang X, Zhao J, Wang S, Sun X, Zhang X, Chen J, Kaplan DL and Zhang Z: Mandibular repair in rats with premineralized silk scaffolds and BMP-2-modified bMSCs. Biomaterials 30: 4522-4532, 2009.

34. Xu XL, Tang T, Dai K, Zhu Z, Guo XE, Yu C and Lou J: Immune response and effect of adenovirus-mediated human BMP-2 gene transfer on the repair of segmental tibial bone defects in goats. Acta Orthop 76: 637-646, 2005.

35. Xiao C, Zhou H, Ge S, Tang T, Hou H, Luo M and Fan X: Repair of orbital wall defects using biocoral scaffolds combined with bone marrow stem cells enhanced by human bone morphogenetic protein-2 in a canine model. Int J Mol Med 26: 517-525, 2010

36. Wozney JM and Rosen V: Bone morphogenetic protein and bone morphogenetic protein gene family in bone formation and repair. Clin Orthop Relat Res: 26-37, 1998.

37. Chen Y, Luk KD, Cheung KM, Xu R, Lin MC, Lu WW, Leong JC and Kung HF: Gene therapy for new bone formation using adeno-associated viral bone morphogenetic protein- 2 vectors. Gene Ther 10: 1345-1353, 2003.

38. Park J, Ries J, Gelse K, Kloss F, von der Mark K, Wiltfang J, Neukam FW and Schneider H: Bone regeneration in critical size defects by cell-mediated BMP-2 gene transfer: A comparison of adenoviral vectors and liposomes. Gene Ther 10: 1089-1098, 2003.
39. Ramezani A, Hawley TS and Hawley RG: Lentiviral vectors for enhanced gene expression in human hematopoietic cells. Mol Ther 2: 458-469, 2000 .

40. Sugiyama O, An DS, Kung SP, Feeley BT, Gamradt S, Liu NQ, Chen IS and Lieberman JR: Lentivirus-mediated gene transfer induces long-term transgene expression of BMP-2 in vitro and new bone formation in vivo. Mol Ther 11: 390-398, 2005.

41. Virk MS, Conduah A, Park SH, Liu N, Sugiyama O, Cuomo A Kang $\mathrm{C}$ and Lieberman JR: Influence of short-term adenoviral vector and prolonged lentiviral vector mediated bone morphogenetic protein- 2 expression on the quality of bone repair in a rat femoral defect model. Bone 42: 921-931, 2008

42. Mehta S and Watson JT: Platelet rich concentrate: Basic science and current clinical applications. J Orthop Trauma 22: 432-438, 2008.

43. Yamada Y,Ueda M, Naiki T, Takahashi M, Hata K and Nagasaka T: Autogenous injectable bone for regeneration with mesenchymal stem cells and platelet-rich plasma: Tissue-engineered bone regeneration. Tissue Eng 10: 955-964, 2004.

44. Oliva A, Passaro I, Di Pasquale R, Di Feo A, Criscuolo M, Zappia V, Della Ragione F, D'Amato S, Annunziata M and Guida L: Ex vivo expansion of bone marrow stromal cells by platelet-rich plasma: A promising strategy in maxillo-facial surgery. Int J Immunopathol Pharmacol 18: 47-53, 2005.

45. Park CG, Joo MW, Jeong J, Kang YK and Lee DR: Evaluation of the effects of the combination of autologous mesenchymal stem cells and platelet-rich plasma on structural bone allograft healing. Cell Tissue Bank 18: 229-238, 2017.

46. Du X, Huang F, Zhang S, Yao Y, Chen Y, Huang H and Bai B: Carboxymethylcellulose with phenolic hydroxyl microcapsules enclosinggene-modified BMSCs for controlled BMP-2 release in vitro. Artif Cells Nanomed Biotechnol 45: 1-14, 2017.

47. Wang SJ, Jiang D, Zhang ZZ, Huang AB, Qi YS, Wang HJ, Zhang JY and Yu JK: Chondrogenic potential of peripheral blood derived mesenchymal stem cells seeded on demineralized cancellous bone scaffolds. Sci Rep 6: 36400, 2016.

48. Livak KJ and Schmittgen TD: Analysis of relative gene expression data using real-time quantitative PCR and the 2(-Delta Delta C(T)) method. Methods 25: 402-408, 2001.

49. Miller L: Analyzing gels and western blots with ImageJ.Lukemiller. org Miscellaneous Topics Vaguely Related to Science, 2010.

50. Chiu LH, Lai WF, Chang SF, Wong CC, Fan CY, Fang CL and Tsai YH: The effect of type II collagen on MSC osteogenic differentiation and bone defect repair. Biomaterials 35: 2680-2691, 2014.

51. Hu Q, Li B, Wang M and Shen J: Preparation and characterization of biodegradable chitosan/hydroxyapatite nanocomposite rods via in situ hybridization: A potential material as internal fixation of bone fracture. Biomaterials 25: 779-785, 2004.

52. Frohbergh ME, Katsman A, Botta GP, Lazarovici P, Schauer CL, Wegst UG and Lelkes PI: Electrospun hydroxyapatite-containing chitosan nanofibers crosslinked with genipin for bone tissue engineering. Biomaterials 33: 9167-9178, 2012.

53. Eppley BL, Woodell JE and Higgins J: Platelet quantification and growth factor analysis from platelet-rich plasma: Implications for wound healing. Plast Reconstr Surg 114: 1502-1508, 2004.

54. Bahmanpour SP, Ghasemi MP, Sadeghi-Naini MM and Kashani IRP: Effects of platelet-rich plasma \& platelet-rich fibrin with and without stromal cell-derived factor-1 on repairing full-thickness cartilage defects in knees of rabbits. Iran J Med Sci 41: 507-517, 2016.

55. Lian Z, Yin X, Li H, Jia L, He X, Yan Y, Liu N, Wan K, Li X and Lin S: Synergistic effect of bone marrow-derived mesenchymal stem cells and platelet-rich plasma in streptozotocin-induced diabetic rats. Ann Dermatol 26: 1-10, 2014.

56. Dong Y, Zhang Q, Li Y, Jiang J and Chen S: Enhancement of tendon-bone healing for anterior cruciate ligament (ACL) reconstruction using bone marrow-derived mesenchymal stem cells infected with BMP-2. Int J Mol Sci 13: 13605-13620, 2012.

57. Itoi T, Harada Y, Irie H, Sakamoto M, Tamura K, Yogo T, Soeta S, Amasaki H, Hara Y and Tagawa M: Escherichia coli-derived recombinant human bone morphogenetic protein-2 combined with bone marrow-derived mesenchymal stromal cells improves bone regeneration in canine segmental ulnar defects. BMC Vet Res 12: 201,2016

This work is licensed under a Creative Commons Attribution-NonCommercial-NoDerivatives 4.0 International (CC BY-NC-ND 4.0) License. 